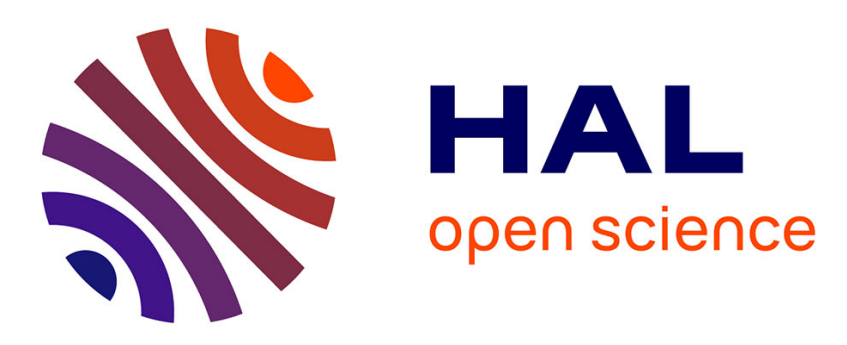

\title{
Description d'un analyseur de rayonnements de thermoluminescence
}

\author{
M. Barland, M. Dailler, H. Darnand
}

\section{To cite this version:}

M. Barland, M. Dailler, H. Darnand. Description d'un analyseur de rayonnements de thermoluminescence. Revue de Physique Appliquée, 1976, 11 (4), pp.527-531. 10.1051/rphysap:01976001104052700 . jpa-00244087

\section{HAL Id: jpa-00244087 https://hal.science/jpa-00244087}

Submitted on 1 Jan 1976

HAL is a multi-disciplinary open access archive for the deposit and dissemination of scientific research documents, whether they are published or not. The documents may come from teaching and research institutions in France or abroad, or from public or private research centers.
L'archive ouverte pluridisciplinaire HAL, est destinée au dépôt et à la diffusion de documents scientifiques de niveau recherche, publiés ou non, émanant des établissements d'enseignement et de recherche français ou étrangers, des laboratoires publics ou privés. 
Classification

Physics Abstracts

0.640

\title{
DESCRIPTION D'UN ANALYSEUR DE RAYONNEMENTS DE THERMOLUMINESCENCE
}

\author{
M. BARLAND, M. DAILLER et H. DARNAND \\ Laboratoire de Spectroscopie et de Luminescence, \\ Université Claude-Bernard, Lyon I, 69621 Villeurbanne, France
}

(Reçu le 22 décembre 1975, accepté le 8 mars 1976)

\begin{abstract}
Résumé. - Le dispositif décrit a été réalisé en vue de l'enregistrement graphique de la composition spectrale de rayonnements de thermoluminescence. Il comprend, en particulier, une mémoire binaire permettant de stocker 32 points de mesure qui correspondent pour le monochromateur, entraîné par un moteur pas à pas, à une excursion de $160 \mathrm{~nm}$ en $1,6 \mathrm{~s}$.

Cet appareil a été appliqué à l'étude du rayonnement de thermoluminescence d'un monocristal de CsI non dopé, mais préalablement chauffé à $820 \mathrm{~K}$ dans un courant d'azote. Il a permis de mettre en évidence que les émissions observées, après excitation par un rayonnement $\mathrm{X}$ à $77 \mathrm{~K}$, sont gaussiennes et que leur largeur à mi-hauteur ainsi que leur position varient avec la température selon des lois qui laissent supposer que le centre d'émission est le même pour les différents pics.
\end{abstract}

\begin{abstract}
An apparatus for recording thermally stimulated luminescence emission spectra is described. It consists of a 32 register binary memory which stores the output of a photomultiplier and a grating monochromator driven by a stepping motor ; $160 \mathrm{~nm}$ are swept in $1,6 \mathrm{~s}$.

This apparatus has been used to analyse the thermally stimulated luminescence emission of a pure CsI crystal heated to $820 \mathrm{~K}$ in $\mathrm{N}_{2}$. After $\mathrm{X}$ irradiation at $77 \mathrm{~K}$, the emission bands which can be observed are gaussian; the variation of their position and half width with temperature indicates that the emission centre is the same for all the peaks.
\end{abstract}

1. Introduction. - L'enregistrement graphique de la composition spectrale d'une émission de thermoluminescence ne peut s'effectuer qu'au moyen d'un dispositif électronique permettant de mettre en mémoire le spectre et de le restituer à une vitesse compatible avec la constante de temps d'un enregistreur à plume. C'est la raison pour laquelle on a cherché à réaliser un appareil de faible prix de revient mais de précision suffisante pour étudier plus particulièrement l'émission de thermoluminescence, après excitation par un rayonnement $X$ à $77 \mathrm{~K}$, d'un monocristal d'iodure de césium $\left({ }^{1}\right)$ non activé mais préalablement chauffé à $820 \mathrm{~K}$ dans un courant d'azote. En effet, à la suite de ce traitement, de même que l'émission bleue de luminescence se trouve accrue [1] la courbe de thermoluminescence présente davantage de pics étroits.

2. Description du dispositif. - Le principe du dispositif d'analyse consiste à associer un photomultiplicateur à un monochromateur à réseau entraîné par un moteur pas à pas. Les rayonnements à étudier pouvant être de relativement faible intensité, on a choisi un

(1) Echantillon fourni par Quartz et silice. monochromateur ouvert à $F / 3,5$ dont la largeur de bande, définie à $0,2 \mathrm{~nm}$ près, peut atteindre $8 \mathrm{~nm}$.

Des enregistrements d'émissions de thermoluminescence, effectués dans le cas de vitesses de réchauffement de quelques dixièmes de degré par seconde, ont permis d'évaluer à deux secondes la durée maximale de l'analyse. Il s'avère en effet que dans ces conditions l'intensité, au passage par son maximum, reste pratiquement constante, même dans le cas de pics dont la largeur à mi-hauteur est voisine de $10 \mathrm{~K}$.

L'étendue du domaine spectral à explorer ayant été fixée à $150 \mathrm{~nm}$, le moteur a été défini de telle sorte qu'un pas corresponde à une augmentation de $5 \mathrm{~nm}$ de la longueur d'onde affichée. La durée d'un pas s'est trouvée être de $20 \mathrm{~ms}$ mais on a observé qu'il fallait attendre encore $20 \mathrm{~ms}$ pour que le réseau du monochromateur soit parfaitement immobile.

La partie électronique du dispositif a été conçue à partir d'une mémoire binaire réalisée en assemblant plusieurs circuits mémoire vive de 64 bits. Les signaux sont prélevés aux bornes d'une résistance de $1 \mathrm{M} \Omega$ traversée par le courant du photomultiplicateur et leur transcription en mots est assurée par un convertisseur analogique digital de 8 bits dont le temps de conversion est inférieur à $1 \mathrm{~ms}$. Compte tenu des intensités de 


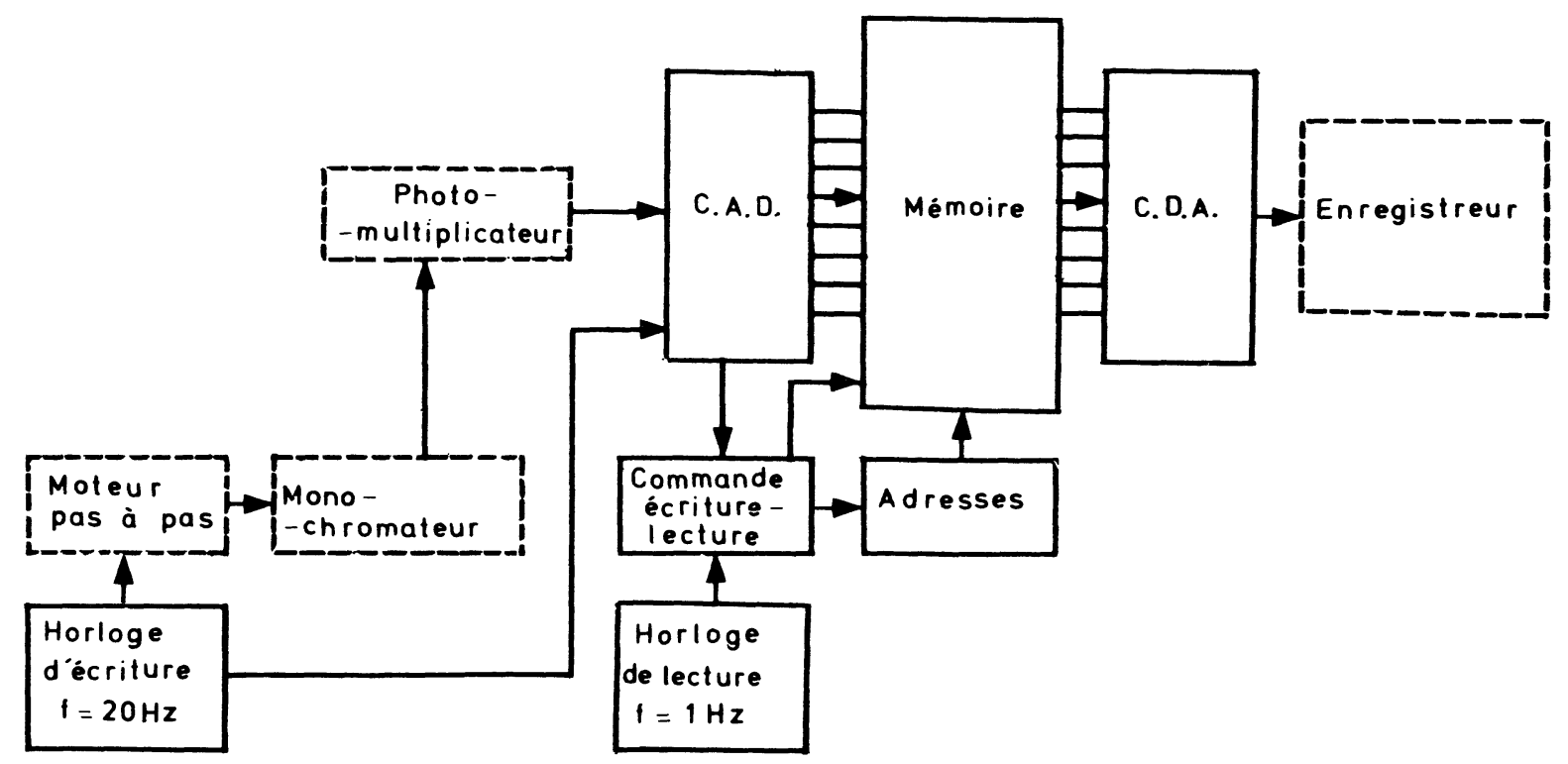

FIG. 1. - Schéma synoptique du dispositif d'analyse.

quelques nA des courants d'obscurité des photomultiplicateurs utilisés et de leur bruit, à chaque bit a été associée une variation du courant de $4 \mathrm{nA}$ de sorte que la valeur maximale des signaux est limitée à $1 \mathrm{~V}$.

L'écriture d'un mot dans la mémoire s'effectue en moins de $1 \mathrm{~ms}$ et a été fixée $40 \mathrm{~ms}$ après le début de chaque pas du moteur qui est commandé par un générateur de fréquence $20 \mathrm{~Hz}$. Ainsi, au cours de chaque analyse dont le déclenchement doit être commandé manuellement au moment où l'émission passe par son maximum, 32 points de mesure correspondant à un domaine spectral exploré de $160 \mathrm{~nm}$ peuvent être enregistrés en 1,6 $\mathrm{s}$ au moyen de quatre circuits mémoire élémentaires. Les spectres obtenus sont conservés dans la mémoire jusqu'à ce qu'il soit procédé à un nouvel enregistrement ou que la tension d'alimentation soit coupée.

La transcription des spectres sur un enregistreur à plume nécessite un convertisseur digital-analogique. On a choisi un circuit modulaire dont le temps de conversion se trouve être de $50 \mu$ s. Compte tenu de la constante de temps de l'enregistreur utilisé, cette transcription est commandée par un générateur de fréquence $1 \mathrm{~Hz}$ et sa durée est de $32 \mathrm{~s}$.

L'ensemble du dispositif dont le schéma synoptique est représenté sur la figure 1 a été réalisé sur circuit imprimé sous forme de cartes enfichables.

3. Etude spectrale du rayonnement de thermoluminescence d'un monocristal de CsI. - Afin de vérifier les performances du dispositif, on a effectué une étude spectrale de l'émission de thermoluminescence d'un monocristal de CsI non activé mais préalablement chauffé à $820 \mathrm{~K}$ dans un courant d'azote et excité par un rayonnement $X(50 \mathrm{kV}, 5 \mathrm{~mA})$ à $77 \mathrm{~K}$. Afin d'obtenir des signaux d'amplitude suffisante, un photomultipli- cateur sensible (E. M. I., 6 256-S) a dû être utilisé. On a alors constaté que, pour une tension de $1300 \mathrm{~V}$, le bruit qui se superpose au courant $I$ du photomultiplicateur introduit une erreur bien supérieure à celle relative à la partie purement électronique du dispositif. Cette erreur dépend bien entendu de l'intensité de $I$, comme le montre le tableau I, et ne peut être réduite

\section{TABLEAU I}

$\begin{array}{cccc}I(\mathrm{nA}) & 800 & 175 & 100 \\ - & - & - & - \\ \begin{array}{c}\text { Précision associée à la par- } \\ \text { tie électronique } / \%\end{array} & 0,5 & 2,2 & 4,0 \\ \text { Précision observée } / \% & 8 & 15 & 17\end{array}$

par intégration si l'on ne veut pas diminuer la vitesse d'enregistrement. Il en résulte une incertitude sur l'intensité des maximums, leur position et leur largeur à mi-hauteur. Afin d'évaluer la précision avec laquelle ces deux derniers paramètres peuvent être déterminés, on a procédé, dans les mêmes conditions, à des analyses répétées de la composition spectrale des maximums de thermoluminescence du monocristal de CsI. Quatre pics ont une intensité suffisante pour permettre une telle étude, il s'agit de ceux qui se situent à $99,126,158$ et 206 K (Fig. 2).

Afin d'obtenir, pour chaque pic, des spectres dont les intensités puissent être comparées en valeur absolue, on a dû effectuer des irradiations $X$ de $6 \mathrm{~h}$, de manière à atteindre l'état de saturation des pièges. Un second photomultiplicateur, qui permet de suivre dans le temps l'émission globale de luminescence, a en effet permis de montrer que, dans ces conditions, l'intensité de chacun des pics reste sensiblement constante au cours des différents essais. Les intensités moyennes ainsi relevées sont 


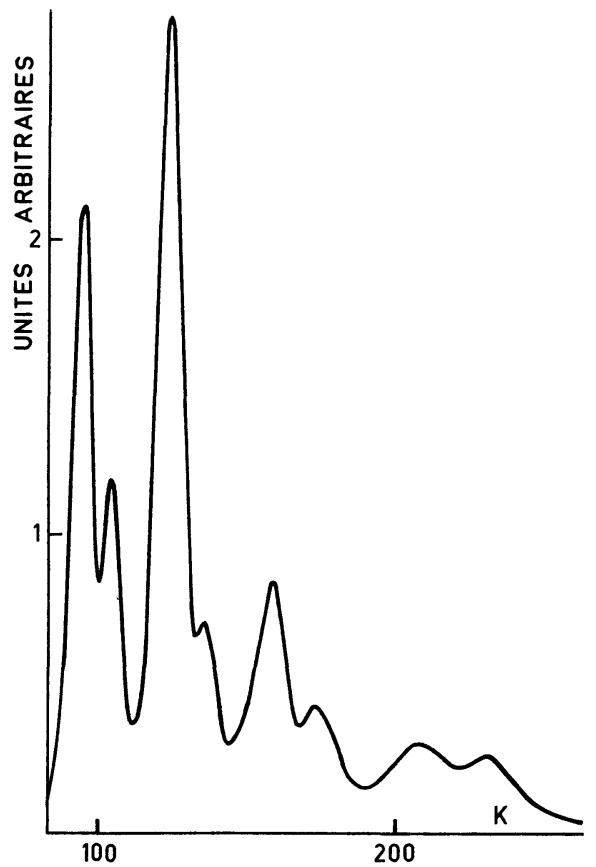

FIG. 2. - Thermoluminescence obtenue après excitation par un rayonnement $\mathrm{X}$ d'un monocristal de CsI préalablement chauffé à $820 \mathrm{~K}$ dans un courant d'azote.

portées dans le tableau II. Les erreurs dont elles sont entâchées proviennent d'ailleurs, vraisemblablement en grande partie, du fait que le photomultiplicateur doit être déplacé pendant les irradiations $X$, alors que l'ensemble du dispositif d'analyse reste en place.

Pour les différents essais, en raison des faibles intensités, la largeur des fentes du monochromateur a été choisie égale à $8 \mathrm{~nm}$ et la vitesse de réchauffement fixée

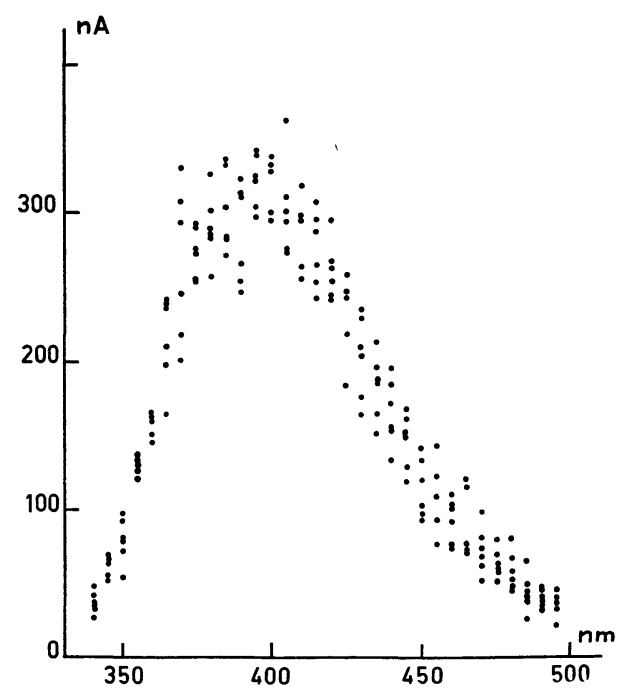

Fig. 3. - Spectre du maximum d'émission de $99 \mathrm{~K}: \bullet$ Points expérimentaux obtenus au cours des différents essais. à $0,7 \mathrm{~K} / \mathrm{s}$. Toutefois, même dans ces conditions, le flux reçu par la photocathode n'atteint pas $10^{-11} \mathrm{~W}$. A titre d'exemple, on a représenté, sur la figure 3, pour le maximum de $99 \mathrm{~K}$, les points correspondant aux répartitions spectrales énergétiques obtenues. En fait, pour analyser l'ensemble des résultats relatifs aux différents pics, on a préféré effectuer, pour chaque bande spectrale, la moyenne arithmétique des intensités relevées lors des analyses répétées d'un même rayonnement. Les bandes d'émission sont alors sensiblement gaussiennes comme le montrent les figures 4 à 7 . Les

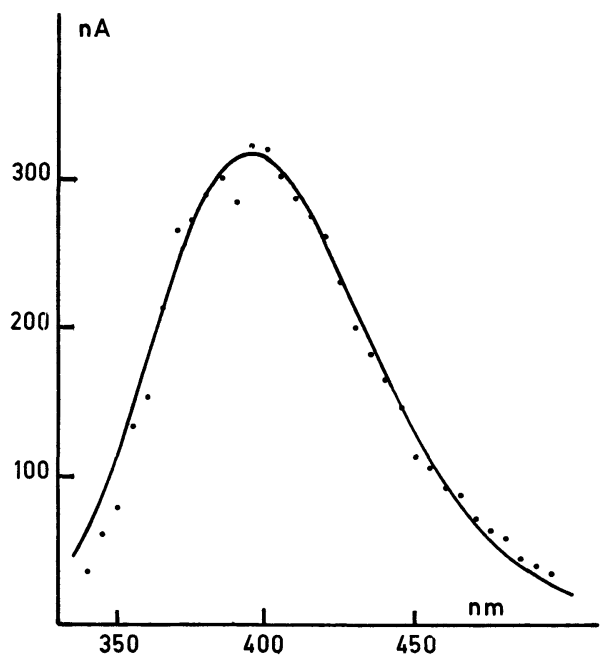

Fig. 4. - Spectre du maximum d'émission de $99 \mathrm{~K}: \bullet$ Valeur moyenne des résultats expérimentaux. - Courbe de Gauss.

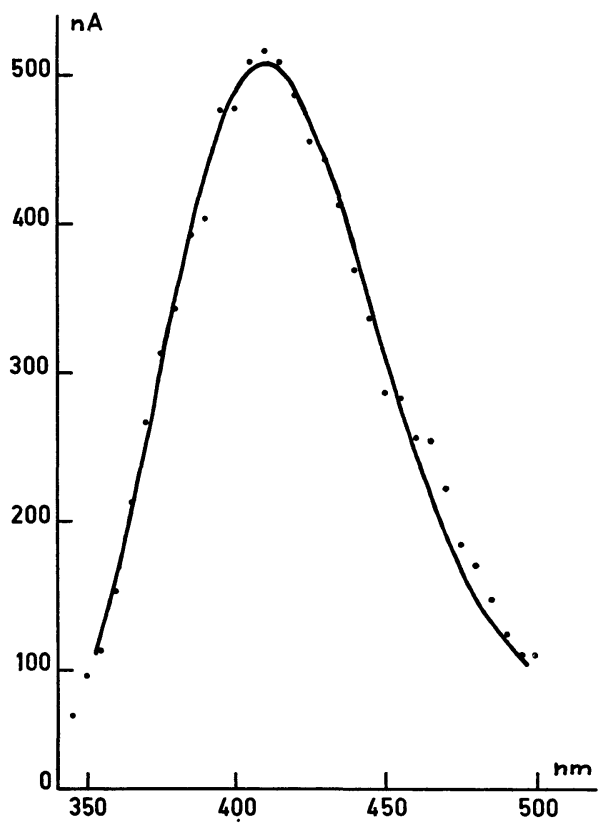

Fig. 5. - Spectre du maximum d'émission de $126 \mathrm{~K}: \bullet$ valeur moyenne des résultats expérimentaux. - Courbe de Gauss.

TABLEaU II

Température (K)

Intensité moyenne (nA)
99

126

$1200 \pm 14 \% \quad 1900 \pm 5 \%$
206

$280 \pm 8 \%$ $210 \pm 11 \%$ 


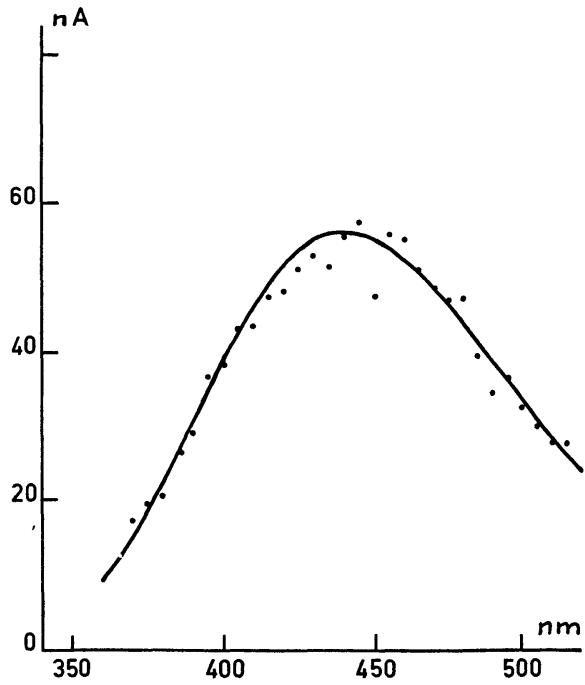

Fig. 6. - Spectre du maximum d'émission de $158 \mathrm{~K}: \bullet$ valeur moyenne des résultats expérimentaux. - Courbe de Gauss.

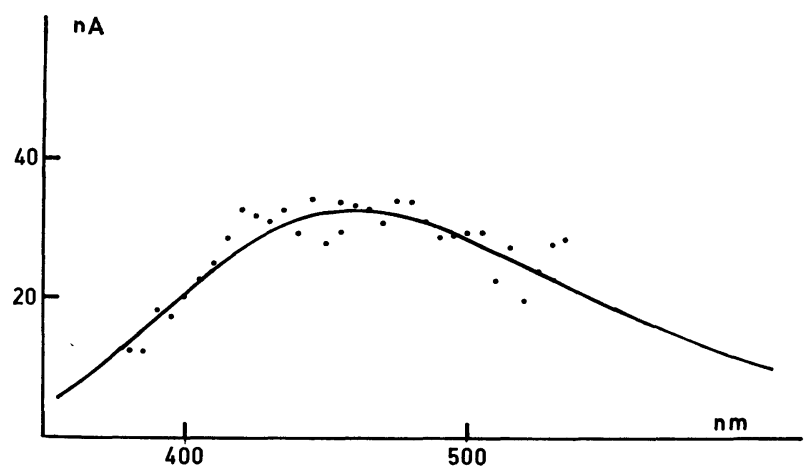

FIg. 7. - Spectre du maximum d'émission de $206 \mathrm{~K}$ : • valeur moyenne des résultats expérimentaux. - Courbe de Gauss.

valeurs obtenues pour l'intensité maximale $I_{\mathrm{m}}$, le nombre d'ondes correspondant $v_{\mathrm{m}}$ et la largeur à mihauteur $2 L_{\mathrm{e}}$ de chaque pic sont portées dans le tableau III. Il apparaît ainsi que plus l'émission analysée se situe à une température élevée, plus son spectre se trouve déplacé du côté des grandes longueurs d'onde. En outre, les rayonnements correspondant aux maximums de 99 et $126 \mathrm{~K}$ sont de plus courte longueur d'onde que ceux observés dans le cas d'un cristal activé par des ions divalents $[2,3]$ ou d'un cristal non activé mais n'ayant subi aucun traitement thermique $\left({ }^{2}\right)$.

(2) Travail en cours.
4. Analyse des résultats. - On a cherché à se rendre compte si les centres responsables de l'émission de thermoluminescence étaient les mêmes pour les quatre pics. Dans ce cas, les valeurs de $v_{\mathrm{m}}$ et de $L_{\mathrm{e}}$ qui figurent dans le tableau III devraient varier avec la température selon les lois :

$$
\begin{aligned}
& v_{\mathrm{m}}(T)=v(0)+A \operatorname{Coth}\left(h v_{\mathrm{e}} / 2 k T\right) \\
& L_{\mathrm{e}}(T)=L_{\mathrm{e}}(0)\left[\operatorname{Coth}\left(h v_{\mathrm{e}} / 2 k T\right)\right]^{1 / 2}
\end{aligned}
$$

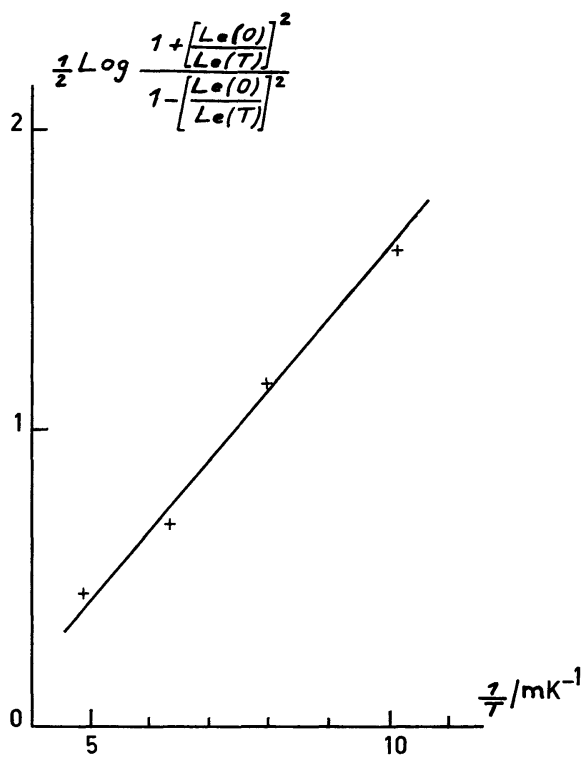

FIG. 8. - Voir texte.

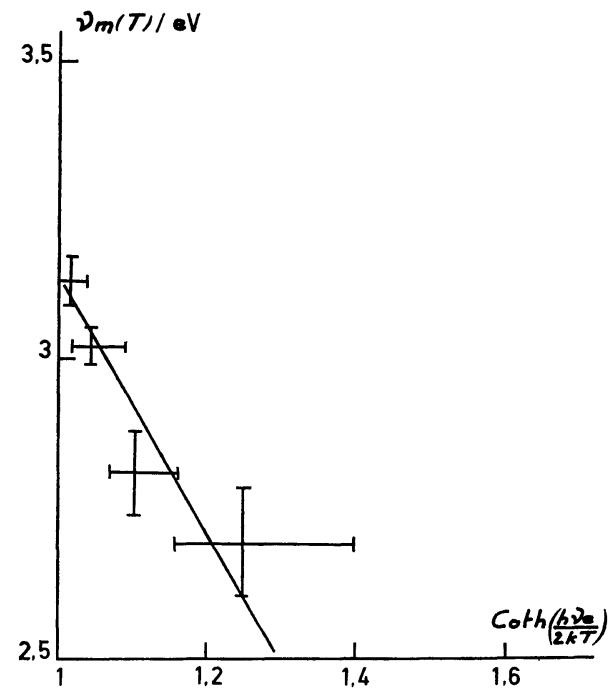

Fig. 9. - Voir texte.

\begin{tabular}{|c|c|c|c|c|}
\hline $\begin{array}{l}\text { Température } \\
\text { (K) }\end{array}$ & $\begin{array}{c}I_{\mathrm{m}} \\
(\mathrm{nA})\end{array}$ & $\mathrm{cm}^{-1}$ & $\mathrm{eV}$ & $2 L_{\mathrm{e}} / \mathrm{eV}$ \\
\hline$\overline{99}$ & 317 & $25320 \pm 320$ & $3,14 \pm 0,04$ & $0,53<0, \overline{, 68}<0,71$ \\
\hline 126 & 507 & $24390 \pm 240$ & $3,02 \pm 0,03$ & $0,59<0,65<0,72$ \\
\hline 158 & 56 & $22730 \pm 520$ & $2,81 \pm 0,07$ & $0,58<0,77<0,97$ \\
\hline 206 & 32 & $21740 \pm 700$ & $2,69 \pm 0,09$ & $0,53<1,01<1,29$ \\
\hline
\end{tabular}

TABLEAU III 
La valeur de $L_{\mathrm{e}}(0)$ ayant été évaluée à $4800 \mathrm{~cm}^{-1}$, il apparaît sur la figure 8 que les points représentant :

$$
\frac{1}{2} \log \frac{1+\left[L_{\mathrm{e}}(0) / L_{\mathrm{e}}(T)\right]^{2}}{1-\left[L_{\mathrm{e}}(0) / L_{\mathrm{e}}(T)\right]^{2}}
$$

en fonction de $1 / T$, se situent effectivement sensiblement sur une droite dont la pente conduit, pour le quantum de vibration $h v_{\mathrm{e}}$, à $0,04 \mathrm{eV}$. Cette valeur a permis de représenter les points d'ordonnée $v_{\mathrm{m}}(T)$ et d'abscisse Coth $\left(h v_{\mathrm{e}} / 2 k T\right)$ et de vérifier qu'ils se situent eux aussi approximativement sur une droite (Fig. 9).

5. Conclusion. - On a réalisé un dispositif de faible prix de revient qui permet, en 1,6 s, d'analyser un rayonnement de thermoluminescence avec une erreur absolue de $4 \mathrm{nA}$; le courant du photomultiplicateur devant être, au plus, égal à 1000 nA. Toutefois, la précision est fortement réduite lorsque, pour des émissions de faible intensité, on doit utiliser un photomultiplicateur de grande sensibilité. Un courant de $100 \mathrm{nA}$ peut alors se trouver entâché d'une erreur atteignant $17 \%$. Cependant, dans ces conditions, il est encore possible d'obtenir des résultats convenables en multipliant les mesures. C'est ainsi que l'on a pu mettre en évidence que la répartition spectrale des pics de thermoluminescence d'un monocristal de CsI, préalablement chauffé à $820 \mathrm{~K}$ dans un courant d'azote, est gaussienne et que le centre d'émission semble être le même pour les quatre pics.

\section{Bibliographie}

[1] Bates, C. W., Hsu, O. L., Salau, A., Spicer, W. E., Phys. Lett. 15A (1975) 425.

[2] Aggerter, M. A., Thèse de Doctorat (Neuchâtel) 1967.

[3] Lamatsch, H., Thèse de Doctorat (Neuchâtel) 1971. 